\title{
An IR-UWB IEEE 802.15.4z Compatible Coherent Asynchronous Polar Transmitter in 28-nm CMOS
}

\author{
Gaurav Singh $^{(}$, Erwin Allebes $\left.{ }^{(}\right)$, Yuming $\mathrm{He}^{(}{ }^{\circledR}$, Evgenii Tiurin ${ }^{(}$, Paul Mateman, \\ Johan F. Dijkhuis, Member, IEEE, Gert-Jan van Schaik, Elbert Bechthum, Johan van den Heuvel, \\ Mohieddine El Soussi, Member, IEEE, Arjan Breeschoten, Hannu Korpela, \\ Gert-Jan Gordebeke ${ }^{\circledR}$, Member, IEEE, Sam Lemey ${ }^{\circledR}$, Member, IEEE, \\ Christian Bachmann, Member, IEEE, and Yao-Hong Liu ${ }^{\circledR}$, Senior Member, IEEE
}

\begin{abstract}
A low-power IEEE 802.15.4z high-rate PHY (HRP) compatible coherent transmitter is described. The proposed transmitter uses a digital polar architecture with fixed amplitude steps in the power amplifier and asynchronous time-discrete pulse shaping. The pulse-shaping unit consists of a finite-impulse response (FIR) filter using current-starved inverter-based delay taps that can be calibrated on-chip. An injection-locked ring oscillator (ILRO)-based frequency synthesis enables wideband operation from 3- to $10-\mathrm{GHz}$ frequency bands. The ILRO also allows for duty-cycled coherent mode operation with 2-4-ns phase locking time and binary phase modulation is applied directly on the oscillator. The on-chip digital front end enables duty cycling (DC) of analog front-end modules with a granularity of 2 ns. Implemented in 28-nm CMOS process, this chip is measured to consume 4.9-mW power in nominal mode with IEEE 802.15.4z high pulse repetition frequency (HPRF) compatible data rate of $6.81 \mathrm{Mb} / \mathrm{s}$ compliant with major spectrum mask regulations for channels 5 and 9. With DC of the oscillator enabled in the energy-efficient mode, a power consumption of $430 \mu \mathrm{W}$ is achieved for packets compatible with legacy pulseposition-modulated IEEE 802.15.4a standard with a data rate of $27.2 \mathrm{Mb} / \mathrm{s}$.
\end{abstract}

Index Terms-Digital transmitter, IEEE 802154a, IEEE $802154 \mathrm{z}$, impulse radio, localization, polar transmitter, transversal filter, ultra-wideband (UWB).

\section{INTRODUCTION}

$\mathbf{U}$ LTRA-WIDEBAND impulse radios have seen resurgence in popularity due to increased demand for applications requiring spatial awareness, such as secure access, indoor

Manuscript received April 19, 2021; revised August 9, 2021 and September 22, 2021; accepted September 26, 2021. This article was approved by Associate Editor Nagendra Krishnapura. (Corresponding author: Gaurav Singh.)

Gaurav Singh, Erwin Allebes, Yuming He, Evgenii Tiurin, Paul Mateman, Johan F. Dijkhuis, Gert-Jan van Schaik, Elbert Bechthum, Johan van den Heuvel, Mohieddine El Soussi, Arjan Breeschoten, Hannu Korpela, and Yao-Hong Liu are with IMEC Netherlands, $5656 \mathrm{AE}$ Eindhoven, The Netherlands (e-mail: gaurav.singh@imec.nl).

Gert-Jan Gordebeke and Sam Lemey are with the IDLab Electromagnetics Group, Department of Information Technology, Ghent University-IMEC, 9052 Ghent, Belgium (e-mail: sam.lemey@ugent.be).

Christian Bachmann is with the Wireless Sensing Department, IMEC Netherlands, 5656 AE Eindhoven, The Netherlands.

Color versions of one or more figures in this article are available at https://doi.org/10.1109/JSSC.2021.3116895.

Digital Object Identifier 10.1109/JSSC.2021.3116895

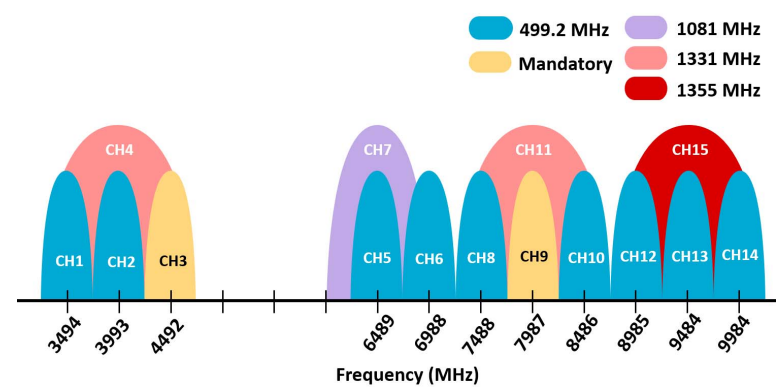

Fig. 1. Channels defined in $802.15 .4 \mathrm{z}$ standard with bandwidths of 500, 1100, and $1310 \mathrm{MHz}$.

localization, asset tracking, augmented reality (AR)/virtual reality (VR), and gaming. Impulse-radio ultra-wideband (IRUWB) employing time-of-flight-based ranging is a promising solution for accurate localization [1].

The recently released IR-UWB IEEE $802.15 .4 \mathrm{z}$ standard mandates coherent mode operation for high-rate PHY (HRP) and enhances the secure ranging operation with a dedicated scrambled timestamp sequence (STS) field in the packet [2]. The use of coherent transceiver improves the link budget of the IR-UWB radio at the cost of additional power dissipation as low-power non-coherent architectures cannot be employed [9]. Furthermore, other low-power IR-UWB transmitter architectures presented in the literature cannot support the coherent modulation requirements of $802.15 .4 \mathrm{z}$ standard [10], [11]. The IEEE $802.15 .4 \mathrm{z}$ standard also proposes two new high pulse repetition frequency (HPRF) modes of 124.8 and $249.6 \mathrm{MHz}$ with higher pulse density compared to the legacy standard. The IEEE $802.15 .4 \mathrm{a} / \mathrm{z}$ standards specify channels from 3.5 to $10 \mathrm{GHz}$ (Fig. 1). Transmission in these frequency channels is subject to regional spectral masks regulations [3].

Conventional IR-UWB transmitters use a baseband pulseshaping filter whose output is upconverted to RF with an IQ mixer and transmitted using a linear power amplifier (PA), as shown in Fig. 2(a). The power dissipation in such transmitters to meet the spectrum mask requirements is high [4], [5]. This limits battery lifetime and, consequently, the applications 


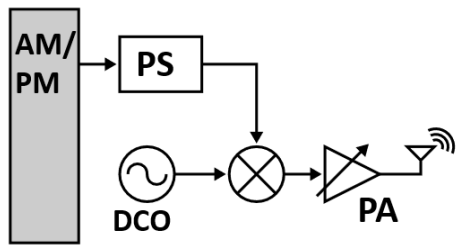

(a)

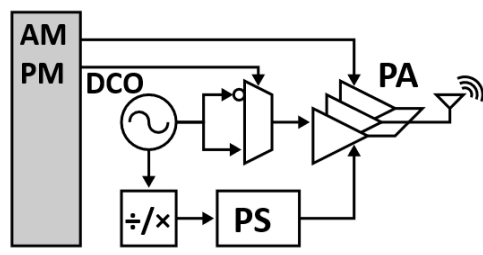

(b)

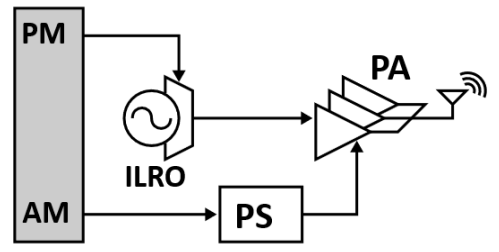

(c)

Fig. 2. (a) IQ-upconversion transmitter. (b) Polar transmitter using the LO clock for synchronization. (c) Proposed asynchronous transmitter architecture.

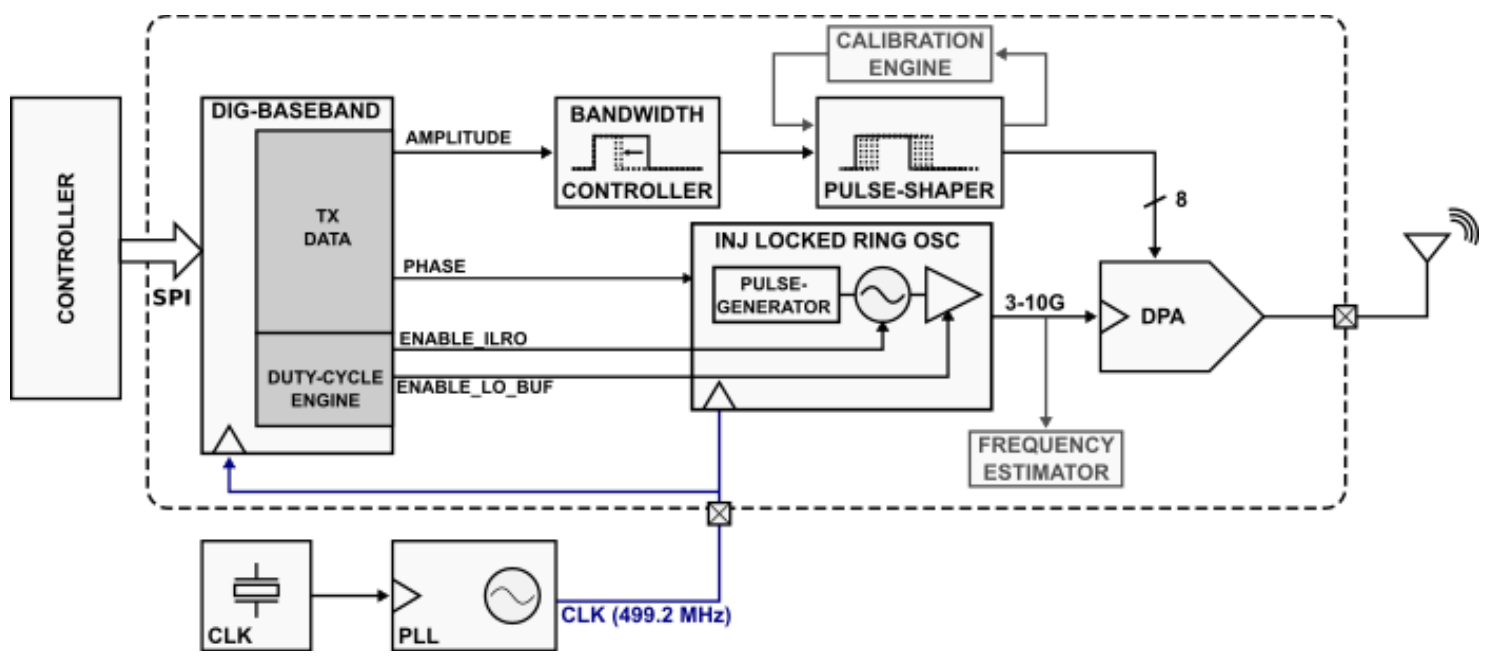

Fig. 3. High-level architecture of the asynchronous polar UWB transmitter IC consisting of a memory-based digital baseband, DC engine, ILRO, pulse shaper, and DPA.

in which IR-UWB can be applied. An alternative solution is to perform the pulse shaping in digital domain and use a polar transmitter by performing a Cartesian to polar transformation. A polar transmitter can employ a non-linear PA leading to high efficiency. However, the Cartesian to polar transformation results in bandwidth expansion, which can result in a clock rate of digital PA (DPA) that is 4-10 times higher than the chip rate resulting in large power dissipation for the overall system. An alternate polar architecture has been proposed in the past where the RF carrier frequency is used as the clock for discretizing the envelope [7]. This is shown in Fig. 2(b) where the digitally controlled oscillator (DCO) output frequency is used as the digital up-sampling clock. The drawback of this approach is that the pulse shape highly depends on the center frequency of the channel.

In this work, we propose an asynchronous polar transmitter employing a pulse shaper that consists of a finite-impulse response (FIR) filter employing current-starved inverter-based delay taps resulting in good power performance tradeoff [see Fig. 2(c)]. The use of an injection-locked ring oscillator (ILRO) for RF carrier generation allows duty cycling (DC) of the DCO and further reduction in power dissipation. The proposed transmitter is compatible with IEEE 802.15.4z standard supporting coherent operation with the lowest power dissipation in the literature known to us. The output spectrum is also compliant with most of regional spectral mask regulations applicable for specific UWB channels.

This article is organized as follows. Section II describes the high-level architecture of the transmitter chip, including an overview of the features of the on-chip digital baseband (DBB). Section III examines the choice of pulse shape and the associated tradeoffs for a power-efficient implementation. Section IV details the key building blocks of the chip. The silicon measurements are discussed in Section V and conclusions provided in Section VI.

\section{ASYNCHRONOUS POLAR TRANSMITTER ARCHITECTURE}

The architecture of the proposed asynchronous polar transmitter integrated circuit (IC) is shown in Fig. 3. An on-chip DBB generates phase and amplitude signals clocked at 499.2 MHz feeding the analog transmitter chain. The analog transmit chain consists of a bandwidth controller, which tunes the pulsewidth of the incoming pulse (nominally $2 \mathrm{~ns}$ ). This is followed by a pulse shaper, which feeds an 8-bit one-hot signal to the DPA. An injection-locked ring oscillator generates the RF carrier and locks to an externally generated $499.2-\mathrm{MHz}$ base clock frequency. The chip includes knobs to support software-based calibration of the ring oscillator frequency as well as the delay taps used in the pulse-shaper block. The chip can be controlled and observed by an external microcontroller over SPI interface.

\section{A. Digital Baseband (DBB)}

The DBB consists of a playback memory unit that can store complete IEEE $802.15 .4 \mathrm{z} / 4 \mathrm{a}$ compatible packets in a compressed format in an on-chip memory. During a packet 


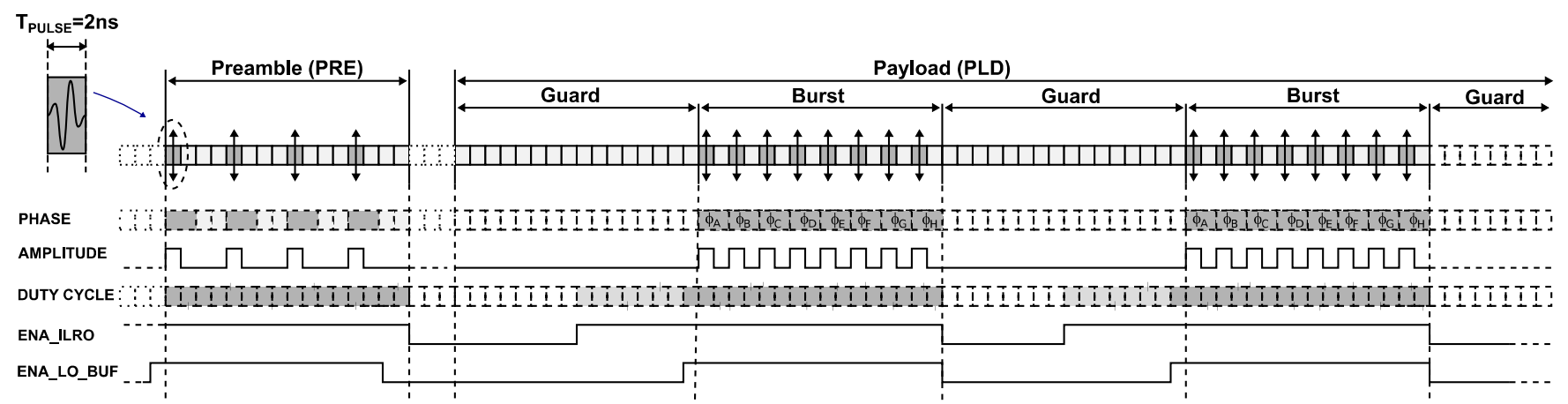

Fig. 4. Timing diagram of preamble and payload parts of 802.15.4z packet with PRF of 124.8 MHz. The DBB outputs phase and amplitude signals along with the enable signals for ILRO and LO buffer on a 2-ns timing grid to control the analog front end.

transmission, the DBB outputs a 2-bit code at $499.2 \mathrm{MHz}$ representing amplitude and phase of the chips needed for binary phase shift keying (BPSK) coded signals. Fig. 4 shows the phase and amplitude signals for a preamble and payload for IEEE 802.15.4z HPRF mode with a 124.8-MHz pulse repetition frequency (PRF). In this mode, each payload bursts consists of alternate chips with a zero and non-zero amplitude. It should also be noted that as shown in Section III, the RF pulse at the output of the transmitter during the payload burst must be longer than $2 \mathrm{~ns}$ to meet the spectrum requirements. This is achieved in the analog front end in pulse-shaper block. For correct operation, the phase must not change, while a pulse is being transmitted. Therefore, the phase bit set during a chip with non-zero amplitude is held constant in the subsequent chip if this subsequent chip has a zero amplitude (Fig. 4).

Along with the amplitude and phase data bits, the DBB also outputs synchronized enable signals for the ILRO, buffers, and PA, which can each be independently controlled with a resolution of 2 ns. This allows DC of each analog block to optimize the overall power dissipation. An example of this shown in Fig. 4, where the ring oscillator is enabled with ENA_ILRO signal eight clock cycles (16 ns) before the beginning of the burst to allow the frequency to settle, while the buffer is enabled just one clock cycle before the burst.

\section{B. Analog Front End}

This transmitter IC supports $500 \mathrm{MHz}$ as well as higher bandwidth channels specified in IEEE 802.15.4z HRP. The support for channels wider than nominal 500-MHz bandwidth is enabled by bandwidth-controller block shown in Fig. 5. The incoming 2-ns square pulse is shrunk to $1 \mathrm{~ns}$ or less and a rectangular output pulse is fed to the pulse-shaper block. Higher transmitter bandwidth in the transmitter can enable improved ranging performance [10].

The pulse-shaper block consists of an FIR filter with eight delay taps. A bus of eight output signals from the pulse shaper is fed to the DPA. The sum of this bus represents the envelope of the output RF signal. The ILRO block generates the RF carrier by locking the phase of a digitally controlled ring oscillator (DCRO) to a reference 499.2-MHz clock. This reference clock is supplied externally with an on-chip clock buffer.

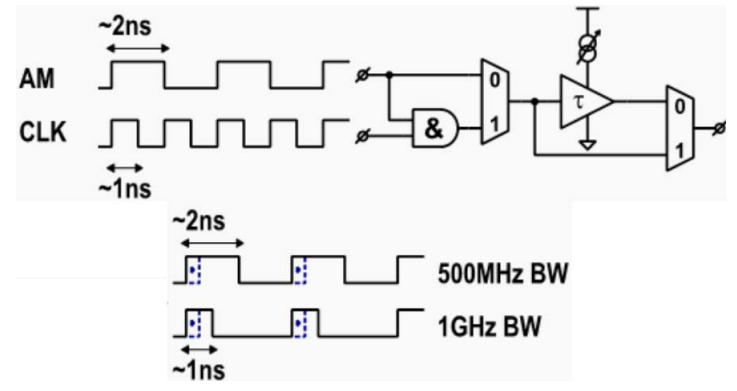

Fig. 5. Conceptual diagram of the bandwidth-controller block.

This pulse-shaper output signals are mixed with the RF carrier in the switched-capacitor-based DPA block. The switched-capacitor implementation allows for summation of pulse-shaper output bus in charge domain to create the desired envelope of the output RF signal to the antenna.

\section{On-Chip Pulse-Shaper Calibration}

To avoid the possibility of incorrect harmonic locking of the ILRO, the open-loop frequency of DCRO must theoretically be within $25 \%$ of the reference clock frequency to the target carrier frequency [13], [14]. Moreover, for the reference spurs due to injection locking to be lower than $-40 \mathrm{dBc}$, the openloop DCO frequency must be within $5 \mathrm{MHz}$ of the target frequency [15]. Therefore, an on-chip frequency-estimation circuit is employed to calibrate the DCRO frequency.

The pulse shaper, which consists of several delay taps, suffers from variations in process, temperature, or other environmental conditions. An on-chip calibration technique is proposed for the delay chain in the pulse-shaper block to calibrate the delays to be within the desired range (detailed in Section IV-B).

\section{Pulse Shape Analysis}

The shape of the pulse in an IR-UWB radio is crucial for ranging as well as for the frequency spectrum of the output to meet the regulatory masks. It can be noted that channels 5 and 9, centered at 6489.6 and $7987.2 \mathrm{MHz}$ can be used in many regions worldwide. However, the regulatory masks pose strict requirements of approximately $-29-\mathrm{dBc}$ suppression of any sidelobes close to the main lobe. The use 


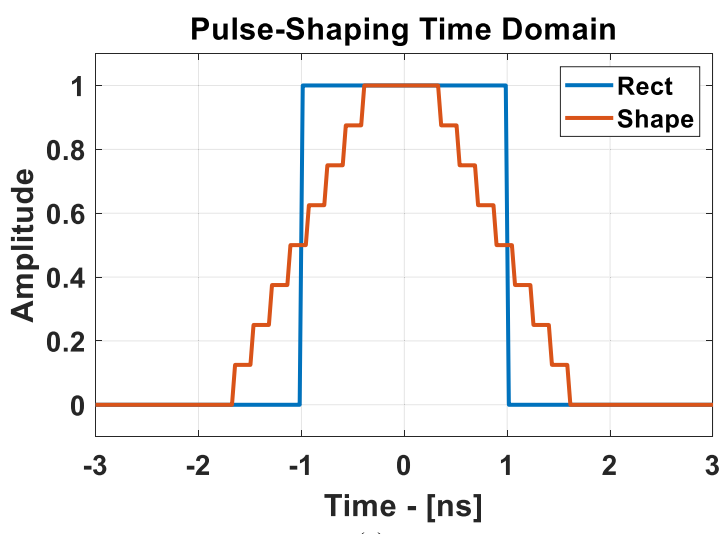

(a)

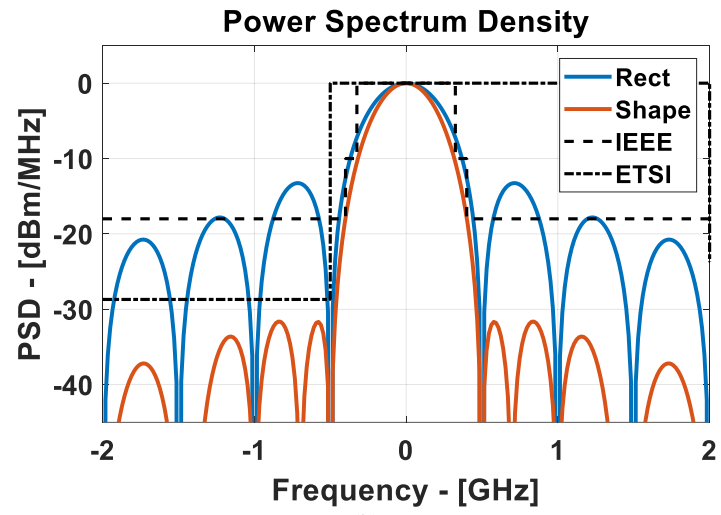

(b)

Fig. 6. (a) Triangular and rectangular pulse shapes in time domain. (b) Normalized close-in frequency spectrum of the corresponding pulse shapes centered with respect to channel $5(6489.6 \mathrm{MHz})$ plotted with a normalized ETSI spectrum mask.

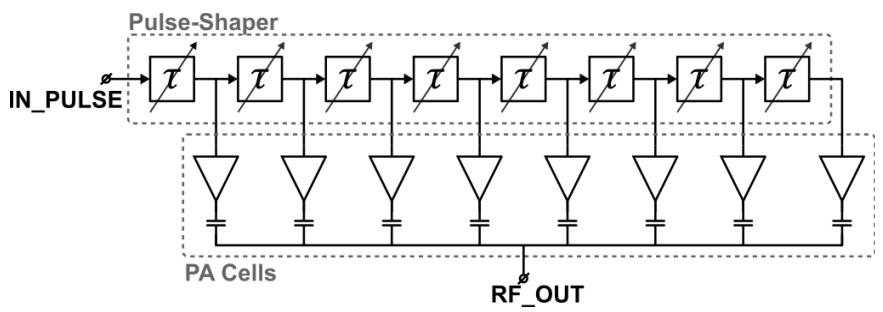

Fig. 7. Conceptual block diagram of the pulse shaping using tunable analog delay cells and switched capacitor PA cells for summation of the delay cell outputs.

of an analog delay line in the FIR filter-based pulse-shaping block allows a power-efficient design while keeping the circuit complexity low.

\section{A. Pulse Shape}

The rectangular pulse shape from the DBB does not have good spectral properties as the sidelobes violate most of the regulatory masks (Fig. 6). A study of pulse shapes with certain constraints has been discussed in [16]. These include triangular, trapezoidal, and other window-shaped pulses. In this analysis, we narrow down the search space to a trapezoidal pulse shape as it allows an energy-efficient design while meeting the regulatory masks for most regions. An major advantage of using trapezoidal pulses is that the desired pulse shape can be generated by a simple summation of outputs of

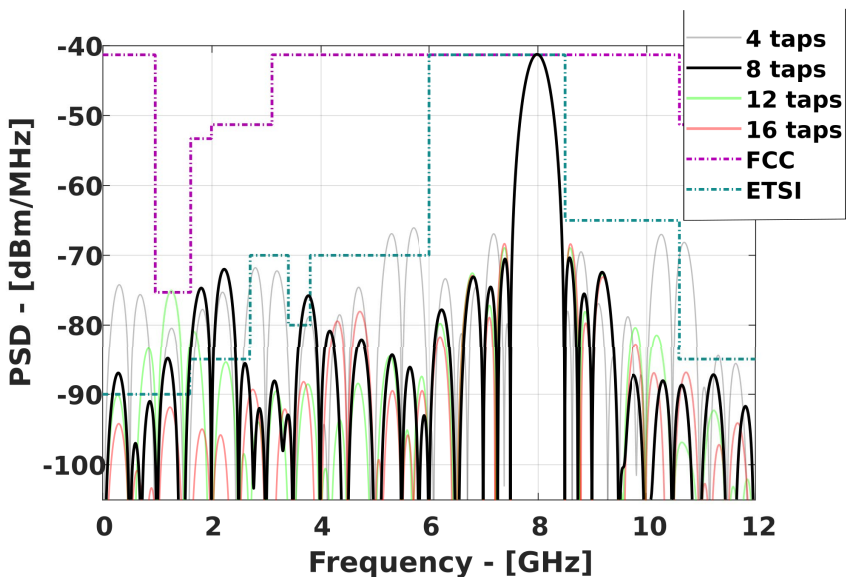

Fig. 8. Frequency spectrum of the pulse with a different number of taps in pulse-shaper delay line.

all the delay taps. The circuit implementation consists of delay taps and switched-capacitor PA cells that summate the output of the filter in charge domain to create the pulse (Fig. 7).

\section{B. Pulse Quantization}

A higher number of delay taps result in lower quantization noise and cleaner frequency spectrum at the expense of higher power dissipation.

Fig. 8 shows the simulated output spectrum as a function of number of delay taps. Although 16 taps result in cleaner spectrum far out from the main lobe, eight taps are sufficient to meet most of the regional spectrum mask regulations for channel 9. The sidelobes at frequencies lower than $4 \mathrm{GHz}$ can easily be filtered by the antenna without requiring additional components, as discussed in Section V.

In this design, we use eight delay taps each connected to a PA cell that is a good tradeoff between spectral purity and design complexity. Each delay tap shifts the incoming pulse by an equal amount of time.

\section{Sensitivity to Variation}

A potential drawback to the proposed approach using asynchronous pulse-shaping approach is the variation in analog delay cells causing the pulse shape and consequently the output to degrade. Fig. 9 shows a plot with sensitivity analysis with $10 \%$ random normal variation in the delay of cells in the pulse-shaper block. It should be noted that the close-in sidelobes are not degraded significantly by mismatch. The variation in far-out sidelobes can be intuitively understood as the delay cells changes have a direct impact on the frequency of the aliases of the spectrum far from the center frequency. The $10 \%$ variation simulated in the delay taps implies that there is sufficient margin in the proposed design with eight delay taps to meet the regulatory spectrum mask requirements.

\section{Transmitter Circuit Design}

\section{A. Injection Locked Ring Oscillator}

Fig. 10 shows the ILRO featuring a pseudo differential DCRO with a frequency operation range of 3-10 GHz. 


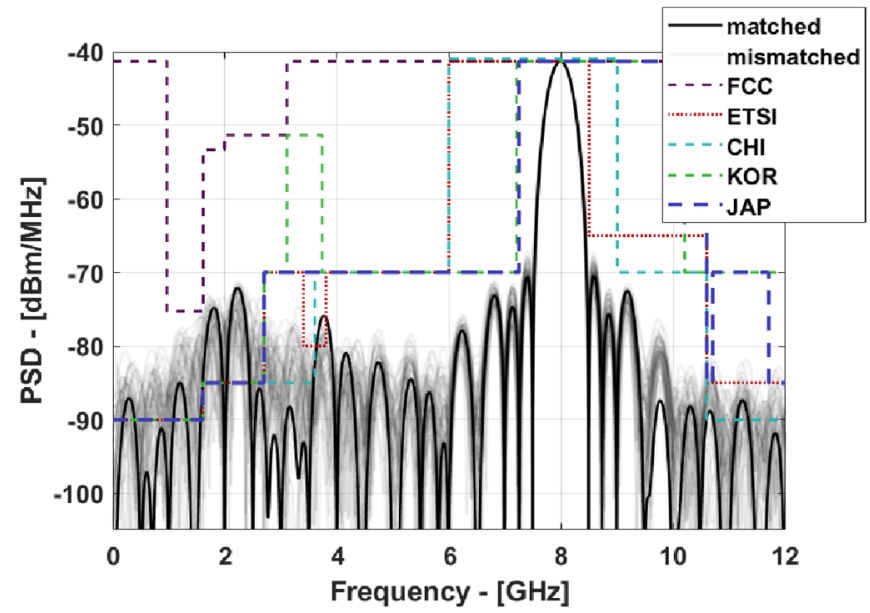

Fig. 9. Output spectrum variations due to random mismatch with standard deviation of $10 \%$ between the eight delay cells.

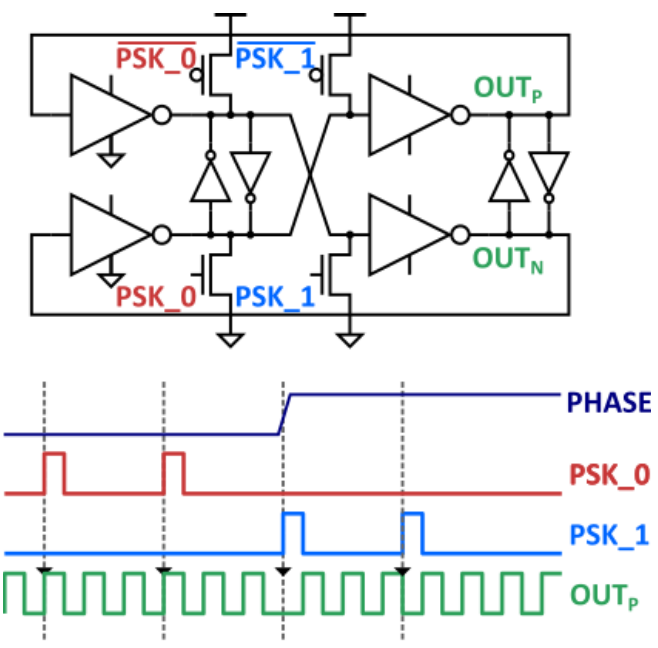

Fig. 10. ILRO circuit with BPSK implemented by reversing the polarity of injection.

The low quality factor of a DCRO allows for fast startup. The DCRO consists of four current-starved inverters and its frequency is tuned by the means of a digitally controlled current digital-to-analog converter (DAC). Before injection locking, the free-running frequency of the DCRO is checked to be calibrated to within $5 \mathrm{MHz}$ of the target frequency using the frequency estimation. If the DCRO is not within this range, a calibration is performed with a software-controlled frequency-locked loop (FLL) using an on-chip frequencyestimation unit. This frequency-estimation unit consists of a counter that measures the number of LO edges in a pre-defined measurement period. The FLL enabled when first using the chip and it is subsequently only needed in case of large frequency drift due to, for instance, temperature drift. A singlefrequency estimation takes $<1 \mu$ s and is a power-efficient way to indicate when frequency drifts greater than the desired limits and an FLL-based calibration is needed.

In the injection-locking mode, the phase of RF carrier in the DCRO is periodically realigned with a clean reference clock edge to suppress the long-term noise from the
DCRO as the noise of the DCRO is effectively high-passfiltered [17], [18]. This feedforward phase-locking technique allows instantaneous phase locking with frequency locked to the intended integer multiple of $499.2-\mathrm{MHz}$ reference clock. The instantaneous phase injection property is exploited for BPSK modulation by direct injection of opposite polarity to the injection nodes of the ILRO, as shown in Fig. 10 [19]. The instantaneous phase locking also enables DC of the ILRO while maintaining coherent mode operation. In this transmitter IC, an option to duty cycle the ILRO and other analog blocks with granularity of a single chip period ( $2 \mathrm{~ns})$ is implemented. The ILRO and the LO buffer can be enabled/disabled with independent scheduling to optimize power dissipation for any packet configuration. Phase lock of the ILRO can be achieved within $16 \mathrm{~ns}$, including the frequency settling time of the DCRO. Therefore, the ILRO is enabled eight clock cycles before the pulse or burst (Fig. 4).

\section{B. Pulse-Shaping Circuits}

The implementation of asynchronous pulse shaper and its interface with the PA is detailed in Fig. 11. The width of the rectangular amplitude pulse is tuned in the bandwidth controller and fed into a seven-tap delay-line-based pulse shaper. Unlike the prior-art synchronous polar techniques [6]-[9], this approach does not rely on the timing grid of the RF carrier. The delay elements of the pulse shaper are implemented using current-starved buffers whose unit delay $\tau$ is programmable by a 4-bit bias current control. This approach lends itself to a compact physical layout of the pulse shaper and PA where the pulse-shaper units can fit close to the PA cells.

An on-chip calibration is scheduled when the transmitter is inactive to account for voltage or temperature variations in the pulse shaper. In this calibration mode, the output of the delay line is fed back to its input converting through a multiplexer converting it into a ring oscillator. A frequency measurement unit is used to measure and calibrate the unit delay $\tau$ by optimizing the pulse-shaper current bias setting. The ability to calibrate the delay taps enables optimization of pulse shape for spectral purity independent of the LO frequency and enables the transmitter to operate in high-bandwidth modes $(>1 \mathrm{GHz})$.

\section{Power Amplifier}

The eight output signals of the pulse shaper are used to enable respective switched-capacitor PA cells (Fig. 11). Each of these cells consists of four unit cells configurable with 2 bits of output power control to meet the power spectral density (PSD) requirement of $-41.3 \mathrm{dBm} / \mathrm{MHz}$ for different PRFs.

The use of switched-capacitor class-D PA unit cells allows for summation of the pulse-shaper outputs in charge domain. However, this structure has a disadvantage in an impulse radio that the common-mode voltage at the output of this PA cell also follows the output pulses, resulting in larger than permitted frequency content at low frequencies close to dc. Adding a tristate mode to the class-D PA cell when no pulse is being transmitted and pre-biasing the output node to the desired common-mode level using a high-bandwidth amplifier 


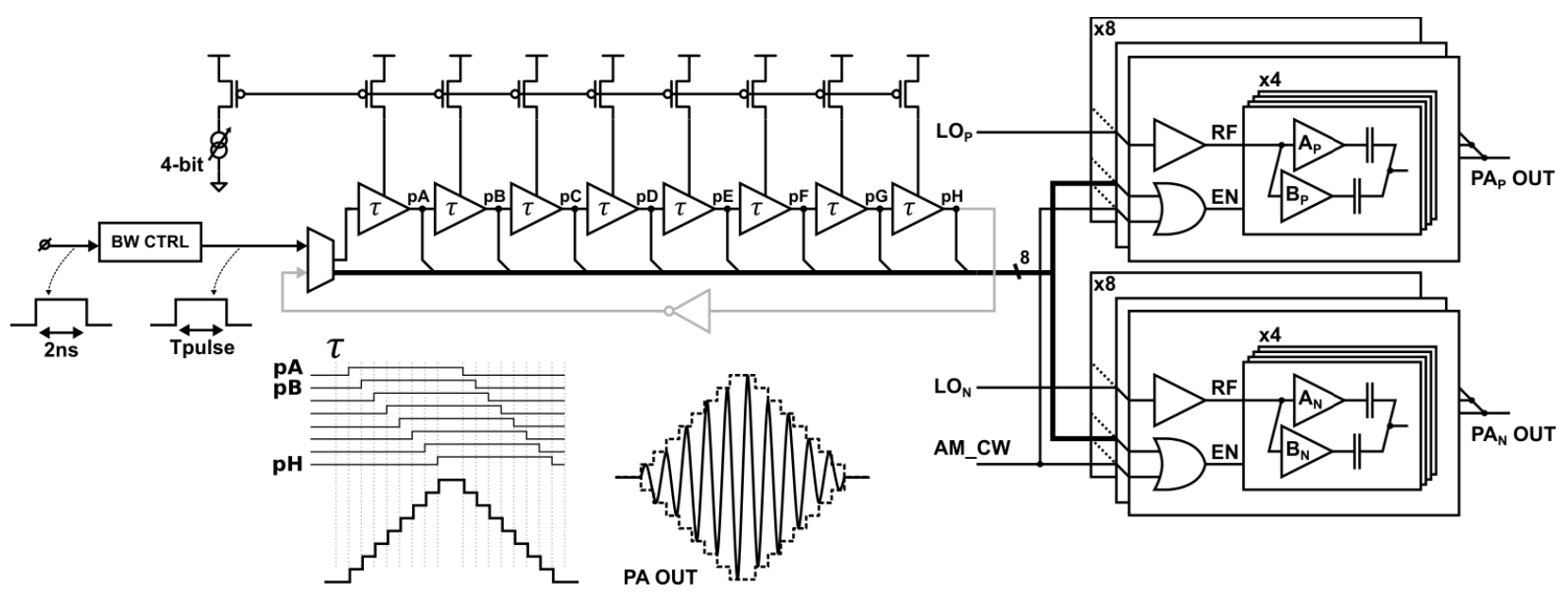

Fig. 11. Pulse-shaping chain includes a bandwidth control block that is used to tune the pulsewidth followed by eight-step pulse-shaping block feeding dual-capacitively coupled PA cells with 2-bit power control.
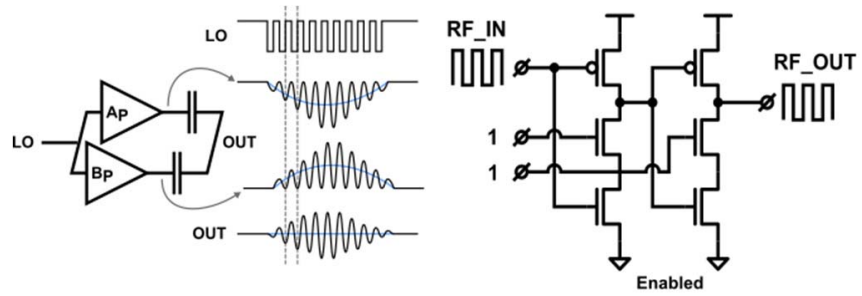

(a)
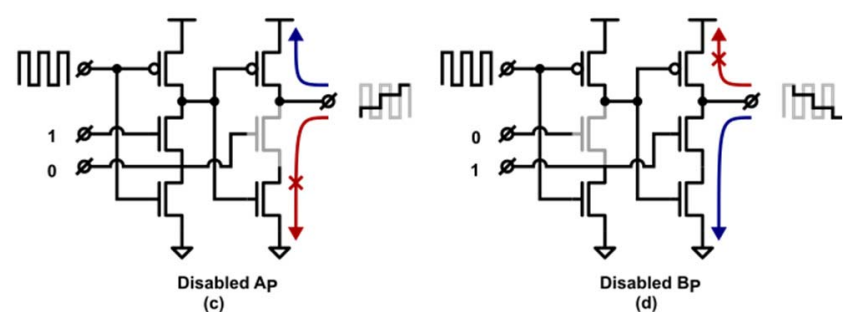

(d)

Fig. 12. (a) Balanced dual capacitively coupled PA cell. (b) Dynamic PA unit cell when enabled. (c) Built-in pull-up logic when disabled for A type unit cell. (d) Built-in pull-down logic when disabled for B type unit cell.

was proposed by de Streel et al. [7]. An alternate approach proposed in [9] is to use a dual-capacitively coupled PA where two PAs cells with tri-state mode are coupled together. When the PA is not transmitting a pulse, the outputs of the two PAs are pulled toward supply and ground with respective switches. The combined output common mode of the overall PA remains close to mid-rail independent of the modulation. This approach is shown to improve the spectrum at low frequencies (close to dc). However, this circuit requires large transistor switches to set the common mode, which must be turned on and off periodically.

In this work, we propose a different approach of implementing the dual-capacitively coupled PA architecture with each PA cell consisting of two units $A_{P}$ and $B_{P}$ with opposite common-mode polarity when disabled (Fig. 12). This results in the output of PA cell being centered around mid-rail similar to [9] without need for large switches to set the common mode of the PA cell. A unit-capacitor size of $160 \mathrm{fF}$ is used in this PA design, which results in small charge/discharge time relative to a pulse duration.

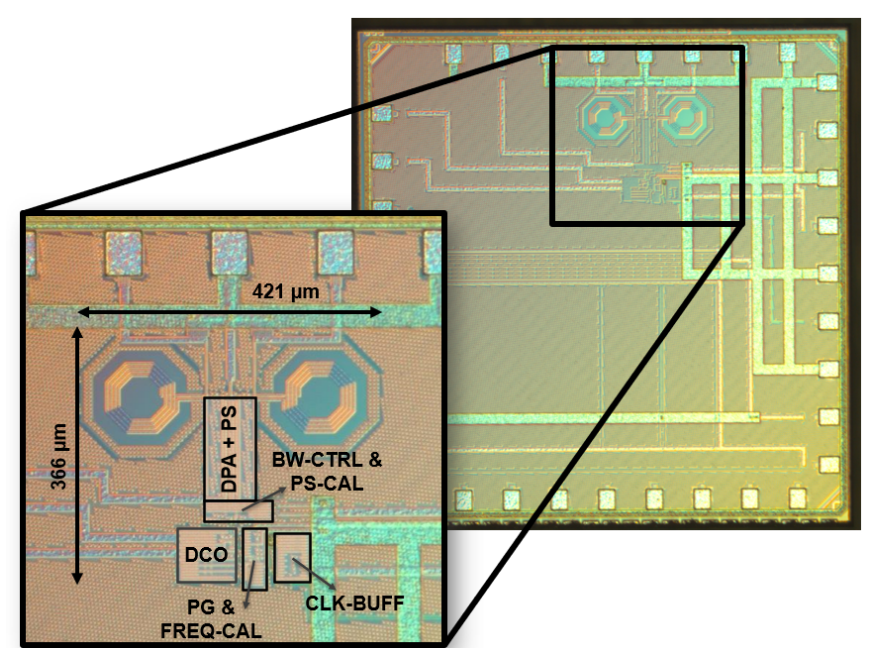

Fig. 13. Chip micrograph of the UWB transmitter IC with an active area of AFE of $0.11 \mathrm{~mm}^{2}$.

\section{Measurement Results}

The chip is implemented in 28-nm CMOS with an active analog front-end area of $0.154 \mathrm{~mm}^{2}$ (Fig. 13) and measured with a supply voltage of $0.9 \mathrm{~V}$. The chip is tested in two different modes: 1) a nominal mode that is compatible with 802.15.4z packets without DC of the ILRO and 2) an efficient mode where the ILRO is duty-cycled while still being compatible with the 802.15.4a standard.

Fig. 14 shows the time-domain plots of $802.15 .4 \mathrm{z}$ preamble and payload in nominal mode. The $802.15 .4 \mathrm{z}$ payload shown here has a PRF of $124.8 \mathrm{MHz}$. The individual pulses are of approximately 4-ns duration for both preamble and payload pulses. In this mode during payload, a chip with zero amplitude between active chips allows the pulses to return-to-zero during phase transition.

We demonstrate an efficient mode operation with DC of DCRO while maintaining coherence. Fig. 15 shows the demodulated amplitude and phase for part of preamble of 802.15.4a with. In this mode, the pulses in the preamble 


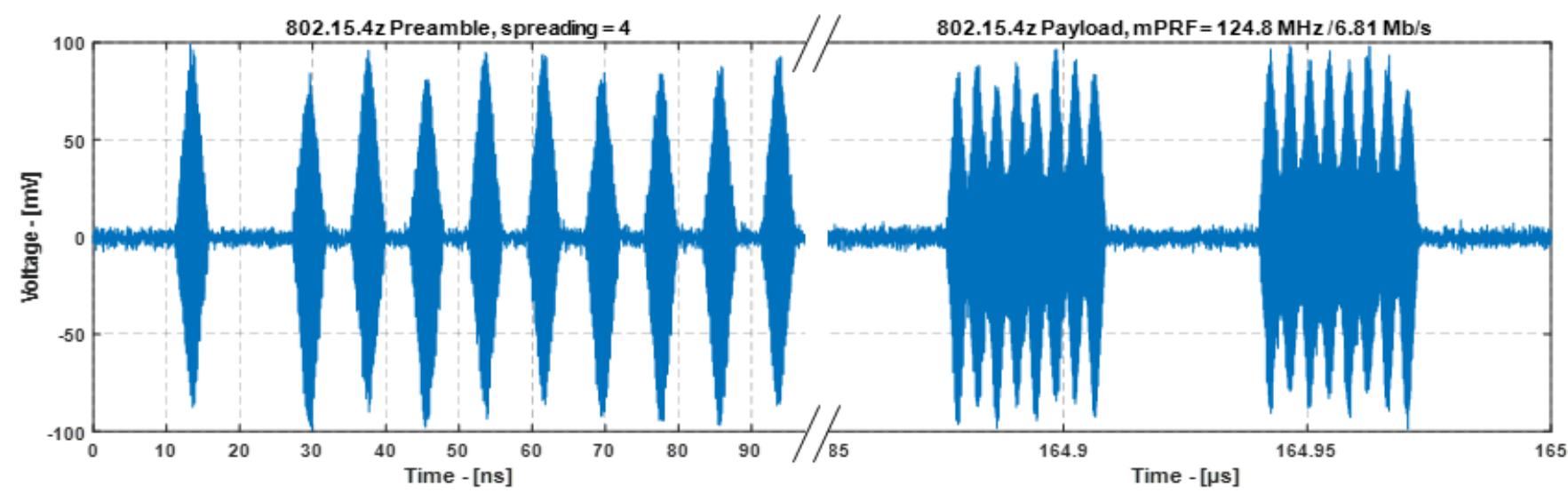

Fig. 14. Time-domain waveforms showing the pulses in $802.15 .4 \mathrm{z}$ preamble and payload mode. It should be noted that the time divisions in the plots for preamble are smaller than payload section.

TABLE I

PERFormanCE SUMmARY AND COMPARISON

\begin{tabular}{|c|c|c|c|c|c|c|c|}
\hline & \multicolumn{2}{|c|}{ This work } & \multirow{2}{*}{$\begin{array}{c}\text { [4] Decawave } \\
\text { DW3000 }\end{array}$} & \multirow{2}{*}{$\begin{array}{c}\text { [5] Decawave } \\
\text { DW } 1000\end{array}$} & \multirow{2}{*}{$\begin{array}{l}{[6] \mathrm{Liu},} \\
\text { RFIC'18 }\end{array}$} & \multirow{2}{*}{$\begin{array}{l}\text { [7] de Streel, } \\
\text { JSSC'17 }\end{array}$} & \multirow{2}{*}{$\begin{array}{l}\text { [9] Mercier, } \\
\text { JSSC'09 }\end{array}$} \\
\hline & Nominal mode & Efficient mode & & & & & \\
\hline Process (nm) & \multicolumn{2}{|c|}{28} & 40 & 90 & 22 & 28 (FDSOI) & 90 \\
\hline Die area $\left(\mathrm{mm}^{2}\right)$ & \multicolumn{2}{|c|}{0.154} & N.A. & N.A. & 0.085 & 0.095 & 0.07 \\
\hline LO freq. (GHz) & \multicolumn{2}{|c|}{$3-10$} & $6.5,8$ & $3.5-6.5$ & $6.5-8$ & $3.5-4.5$ & $2.1-5.7$ \\
\hline IEEE standard & $802.15 .4 \mathrm{z} / 4 \mathrm{a}$ & $802.15 .4 \mathrm{a}$ & $802.15 .4 \mathrm{z}$ & $802.15 .4 \mathrm{a}$ & $802.15 .4 \mathrm{a}$ & $802.15 .4 \mathrm{a}$ & $802.15 .4 \mathrm{a}$ \\
\hline DCO Duty cycling & No & Yes & No & No & N.A. & N.A. & N.A. \\
\hline $\begin{array}{l}\text { UWB indoor } \\
\text { regulations }\end{array}$ & $\begin{array}{c}\mathrm{FCC} / \mathrm{ETSI} / \\
\mathrm{KCC} / \mathrm{JPY} / \mathrm{CHI} \\
\end{array}$ & FCC/ETSI & $\begin{array}{c}\text { FCC/ETSI/ } \\
\text { KCC/JPY/CHI }\end{array}$ & $\mathrm{FCC} / \mathrm{ETSI}$ & $\mathrm{FCC}$ & FCC & FCC \\
\hline $\begin{array}{l}\text { Sidelobe PSD ( dBr) } \\
\text { @ } 500 \mathrm{MHz} \text { offset }\end{array}$ & $-28^{*},-32^{* *}$ & -19 & $-28^{\dagger}$ & $-30 \sim-33^{\dagger}$ & $-11 \sim-13^{\dagger}$ & $-20 \sim-22^{\dagger}$ & $-20 \sim-22 \dagger^{\dagger}$ \\
\hline LO RMS jitter ( ps) & \multicolumn{2}{|c|}{$0.34^{\dagger \dagger}$} & N.A. & N.A. & 9 & N.A. & N.A. \\
\hline CW TX $P_{\text {OUT }}(\mathrm{dBm})$ & \multicolumn{2}{|c|}{$-2.5^{*},-0.7^{* *}$} & N.A. & N.A. & -1 & N.A. & 2.5 \\
\hline Active power $(\mu \mathrm{W})$ & $4900^{+\dagger}$ & $435^{\dagger \dagger}$ & 83800 & 168300 & 300 & 380 (SoC: 650 ) & 4360 \\
\hline $\begin{array}{c}\mathrm{E}_{\mathrm{VDD}} / \mathrm{bit}(\mathrm{pJ} / \mathrm{bit}) \\
@ \text { DDatarate }\end{array}$ & $\begin{array}{c}720 \\
\text { (a) } 6.8 \mathrm{Mb} / \mathrm{s} \\
\end{array}$ & $\begin{array}{c}16 \\
(@ 27.2 \mathrm{Mb} / \mathrm{s} \\
\end{array}$ & $\begin{array}{c}12305 \\
\text { @ } 6.8 \mathrm{Mb} / \mathrm{s}\end{array}$ & $\begin{array}{c}24700 \\
\text { @ } 6.8 \mathrm{Mb} / \mathrm{s}\end{array}$ & $\begin{array}{c}2700 \\
@ 0.11 \mathrm{Mb} / \mathrm{s}\end{array}$ & $\begin{array}{l}14(24 \mathrm{SoC}) \\
\text { @ } 27.2 \mathrm{Mb} / \mathrm{s}\end{array}$ & $\begin{array}{c}280 \\
\text { (a) } 15.6 \mathrm{Mb} / \mathrm{s}\end{array}$ \\
\hline Avg $E_{\text {OUT }} /$ pulse $(p J){ }^{\#}$ & \multicolumn{2}{|c|}{$1.12^{*}, 1.7^{* *}$} & N.A. & N.A. & 1.58 & 0.62 & 1.12 \\
\hline $\begin{array}{c}\mathrm{E}_{\mathrm{VDD}} / \mathrm{pulse}(\mathrm{pJ} / \text { pulse }) \\
@ \mathrm{PRF} \\
\end{array}$ & $\begin{array}{c}39.5 \\
\text { @ } 124.8 \mathrm{MHz} \\
\end{array}$ & $\begin{array}{c}27 \\
\text { (a) } 15.6 \mathrm{MHz} \\
\end{array}$ & $\begin{array}{c}197 \\
\text { (a) } 62.4 \mathrm{MHz} \\
\end{array}$ & $\begin{array}{c}395 \\
\text { (a) } 62.4 \mathrm{MHz} \\
\end{array}$ & $\begin{array}{c}170 \\
\text { (a) } 15.6 \mathrm{MHz} \\
\end{array}$ & $\begin{array}{c}24 \\
\text { (a) } 15.6 \mathrm{MHz} \\
\end{array}$ & $\begin{array}{c}280 \\
\text { (a) } 15.6 \mathrm{MHz}\end{array}$ \\
\hline TX Efficiency \#\# & $4.3 \%$ & $6.2 \%$ & & & $0.9 \%$ & $2.5 \%$ & $0.4 \%$ \\
\hline
\end{tabular}

${ }^{*}$ Channel 9, ${ }^{* *}$ Channel 5, ${ }^{\dagger}$ Estimated from the figures in documentation, ${ }^{\dagger \dagger}$ Reference clock (499.2MHz) from external source,

\# Approximated based on bandwidth of $500 \mathrm{MHz}, \quad$ \# Tx Efficiency $=(\mathrm{E}$ out $/$ pulse $) /\left(\mathrm{E}_{\mathrm{VDD}} / \mathrm{pulse}\right)$

symbol are spread by $128 \mathrm{~ns}$ and the ILRO is enabled only $16 \mathrm{~ns}$ before the pulse, which is sufficient for frequency settling and injection locking of the phase. LO buffer and PA are enabled only $2 \mathrm{~ns}$ before the pulse is transmitted to further optimize the power dissipation. The demodulated phase plot shows correctly demodulated BPSK code. This underlines the benefit of injection locking for DC of the DCRO for use in a coherent receiver.

The on-chip calibration of pulse shape is done by converting the delay line into a ring oscillator and measuring its frequency. The resulting delay as measured with on-chip digital circuitry is shown in Fig. 16(a) as a function of 4-bit control. 

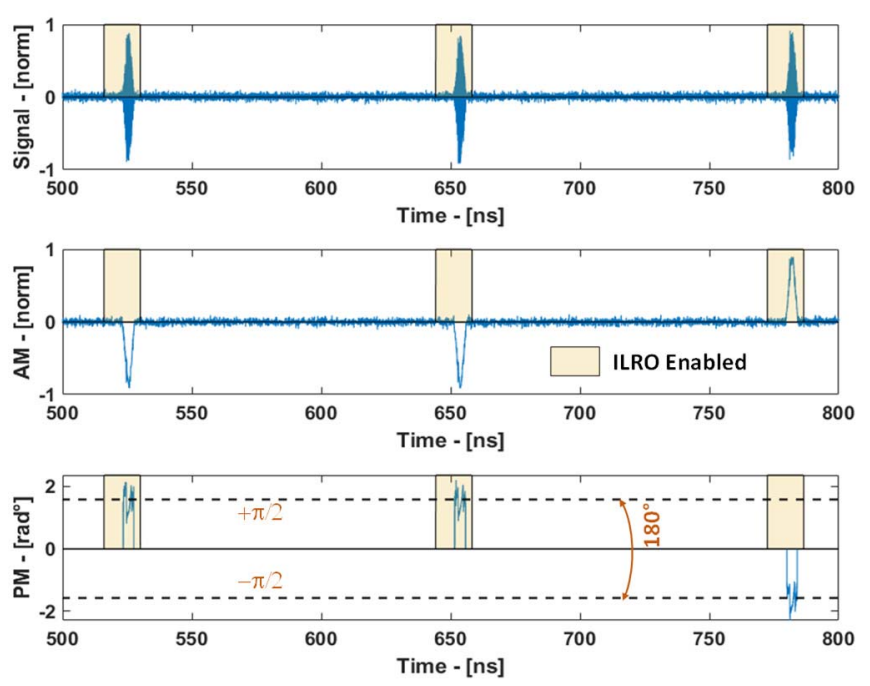

Fig. 15. Demodulated amplitude and phase with DC of the ILRO enabled $16 \mathrm{~ns}$ before the beginning of the pulse with BPSK with injection.

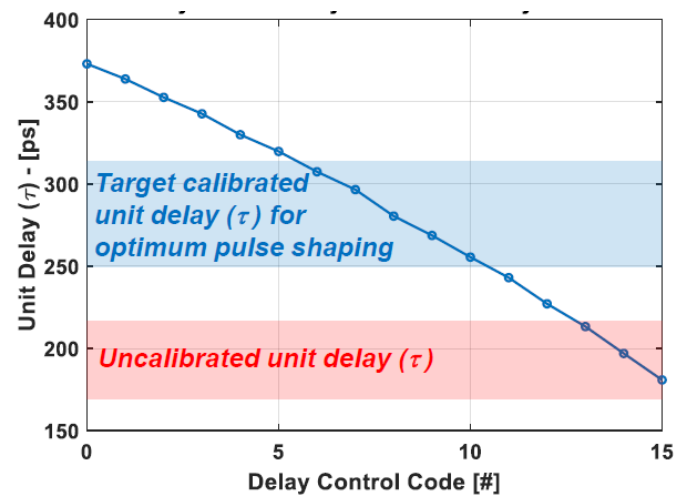

(a)

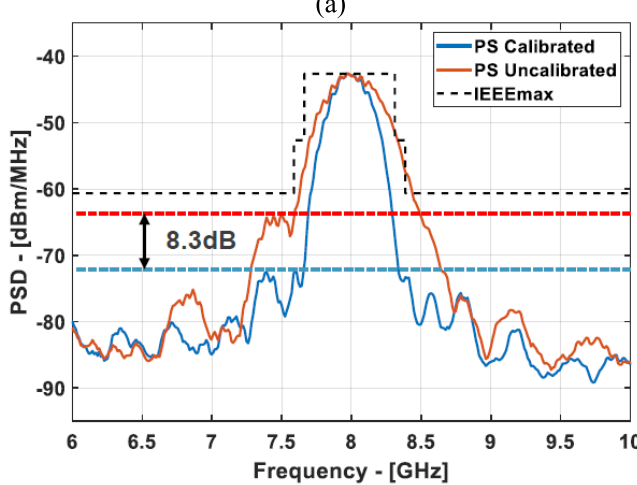

(b)

Fig. 16. (a) Measured unit delay of delay taps in the pulse shaper as a function of 4-bit code. (b) Result of calibration.

Fig. 16(b) shows the resulting spectrum with calibrated and un-calibrated settings of the pulse shape. The delay line can be calibrated as discussed in Section IV-C and allows the first sidelobe close to the main lobe to be lowered by $8.3 \mathrm{~dB}$. The on-chip calibration proposed in this work does not need measurement of the spectrum to calibrate the pulse shape.

Channels 1, 5, 9, and 14 are 500-MHz bandwidth with pulse-shaper calibration, as shown in Fig. 16. The bandwidth controller and pulse-shaper blocks are set in high-bandwidth

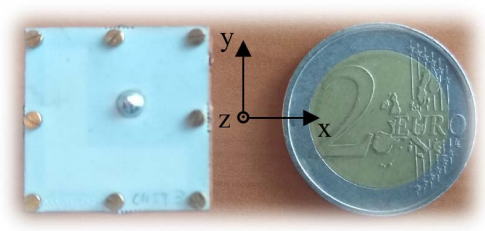

(a)

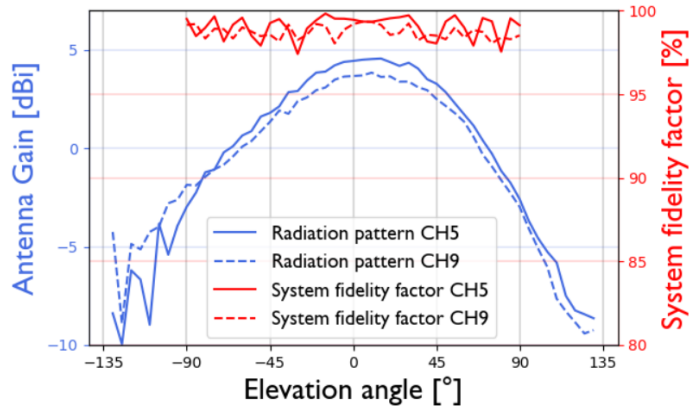

(b)

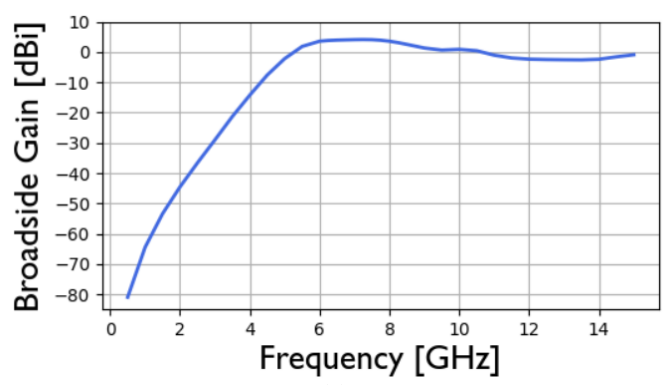

(c)

Fig. 17. (a) Dual-band quarter-mode cavity-backed AFSIW antenna. (b) Measured radiation pattern and SFF for channels 5 and 9 in the $X Z$ plane. (c) Antenna gain as a function of frequency.

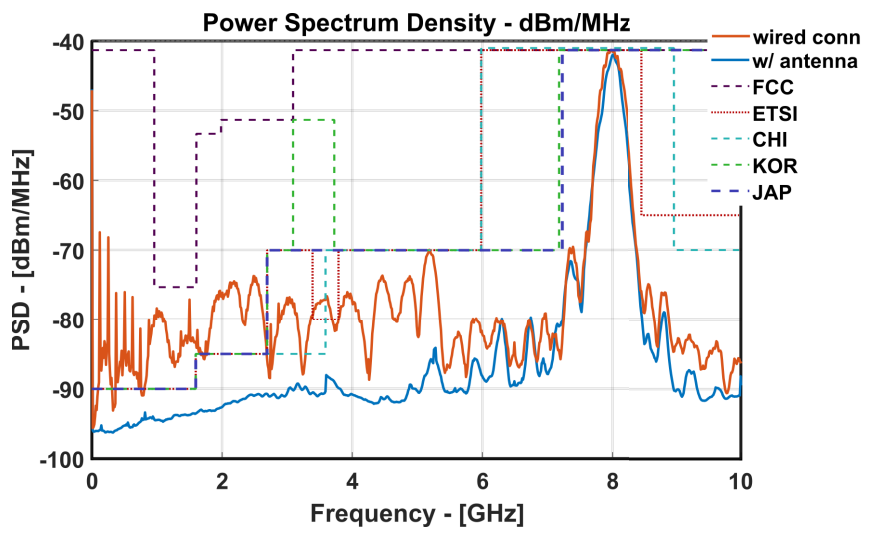

Fig. 18. Measured output spectrum of the transmitter set to channel 9 with wired connection and with antenna.

mode for measurement of channels 4, 7, and 11. The frequency spectrum measurements show compliance with FCC mask, except for some artifacts measured between 1 and $2 \mathrm{GHz}$, which can easily be filtered by an antenna or an off-chip filter.

To exploit the compactness and power efficiency of the IR-UWB transmitter, a quarter-mode cavity-backed slot antenna is implemented in air-filled substrate-integratedwaveguide (AFSIW) technology [Fig. 17(a)]. The AFSIW technology allows a very high radiation efficiency over a wide bandwidth while remaining compatible with standard printed 


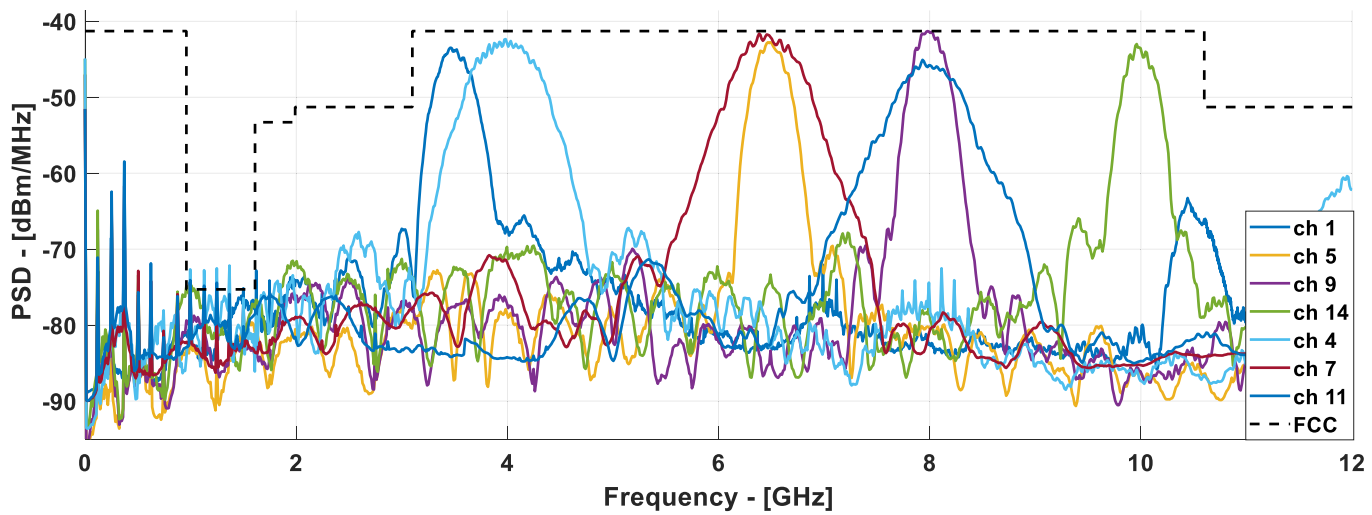

Fig. 19. Measured output spectrum for selected 500-MHz channels-channels 1, 5, 9 and 14-and high-bandwidth channels-channels 4, 7, and 11-along with FCC regulatory mask. The measurements are done with a spectrum analyzer setup as specified by FCC regulations.

circuit board (PCB) technology [20]. Quarter-mode miniaturization is then leveraged to reduce the footprint by a factor four [21]. The proposed antenna covers both channels 5 and 9 with a radiation efficiency over $90 \%$ in each channel, within a footprint of $18 \mathrm{~mm} \times 18 \mathrm{~mm}$. Moreover, through rigorous optimization in the frequency domain and on a system level, a large half-power beamwidth (HPBW) in both channels is obtained in which pulse distortion is minimized by maximizing the system fidelity factor (SFF) [22]. Fig. 17(b) shows the measured radiation pattern at the center frequency of both channels, along with the measured SFF. An HPBW of $110^{\circ}$ is obtained in both channels, with a peak gain of $4.8 \mathrm{dBi}$ in channel 5 and $3.9 \mathrm{dBi}$ in channel 9. An SFF larger than $95 \%$ is achieved indicating minimal pulse distortion. Finally, Fig. 17(c) shows the broadside gain of the antenna as a function of frequency, demonstrating stable antenna gain within channels 5 and 9, and a 20-dB attenuation at frequencies below $4 \mathrm{GHz}$ filtering the transmitter output spectrum at low frequencies.

UWB transmission in channel 9, centered at $7.9872 \mathrm{GHz}$, is allowed by most spectrum regulations worldwide. The EIRP generally permitted for this channel is $-41.3 \mathrm{dBm} / \mathrm{MHz}$. The transmitter described in this article can transmit a continuous wave $(\mathrm{CW})$ tone at a peak output power of $-2.5 \mathrm{dBm}$ (and $-0.7 \mathrm{dBm}$ ) at the UWB channel 9 (and channel 5). When transmitting over a $500-\mathrm{MHz}$-wide channel, this peak output power translates to a PSD of $-29.3 \mathrm{dBm} / \mathrm{MHz}$. The HPRF mode of $124.8 \mathrm{MHz}$ specified in $802.15 .4 \mathrm{z}$ standard is four times lower than the chip rate of $499.2 \mathrm{MHz}$. The maximum PSD supported by this chip in $124.8-\mathrm{MHz}$ PRF mode is -33.8 and $-35.6 \mathrm{dBm} / \mathrm{MHz}$ for channels 5 and 9, respectively. The PA output power can be tuned in steps of $\sim 3 \mathrm{~dB}$ to meet the spectrum regulations. In addition, the PRFs of 62.4, 15.6, and 3.9 MHz are also supported by the transmitter with maximum PSD proportionally lower with respect to the 124.8$\mathrm{MHz}$ PRF mode.

The measured spectrum of the transmitter IC for channel 9 centered at $8 \mathrm{GHz}$ is shown in Fig. 18 with wired and wireless connection. The measured spectrum with wired connection shows some of the artifacts violating some regulatory masks below $4 \mathrm{GHz}$. However, as discussed earlier, these are filtered
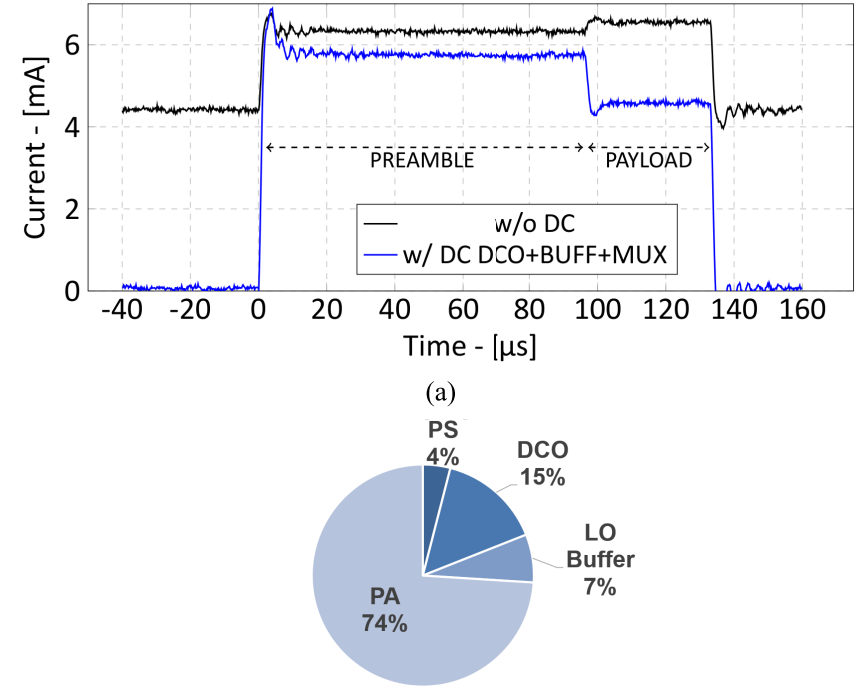

(b)

Fig. 20. (a) Current consumed during packet transmission with and without DC. (b) Power distribution in various blocks of the transmitter with duty-cycle disabled.

by the antenna without need for other external components and the measurement with antenna is compliant with all major regulatory masks. Fig. 19 shows the overlayed measured results for selected UWB channels between 3 and $10 \mathrm{GHz}$ as specified in [2]. This shows compliance with FCC mask for all the channels except for the clock spurs of the $125-\mathrm{MHz}$ onchip digital clock. These low-frequency artifacts can be filtered by an off-chip filter or by the antenna. Fig. 20 shows the power dissipation breakdown of the transmitter. In the nominal mode, the majority of the power is consumed by the PA. The performance of this work in nominal and efficient transmitter mode is summarized and compared with the state of the art in Table I.

\section{CONCLUSION}

This article presents an asynchronous polar transmitter compatible with IEEE 802.15.4z standard supporting coherent channels 5 and 9. The IC fabricated in TSMC 28-nm CMOS technology occupies an active area of $0.154 \mathrm{~mm}^{2}$. With asyn- 
chronous pulse-shaping and fine-grained DC, this chip demonstrates the lowest power $802.15 .4 \mathrm{z}$ compatible transmitter and with minimal power overhead compared to previously reported 802.15.4a transmitters.

\section{REFERENCES}

[1] S. Gezici and H. V. Poor, "Position estimation via ultra-wide-band signals," Proc. IEEE, vol. 97, no. 2, pp. 386-403, Feb. 2009, doi: 10.1109/JPROC.2008.2008840.

[2] IEEE Standard for Low-Rate Wireless Networks-Amendment 1: Enhanced Ultra-Wideband (UWB) Physical Layers (PHYs) and Associated Ranging Techniques, IEEE Standard 802.15.4z-2020, (Amendment to IEEE Std 802.15.4-2020), Aug. 25, 2020, pp. 1-174, doi: 10.1109/IEEESTD.2020.9179124.

[3] Short Range Devices (SRD) Using Ultra Wide Band (UWB); Part 3. Worldwide UWB Regulations Between 3,1 and 10,6 GHz, document ETSI TR 103 181-3-V2.1.1, 2019.

[4] (2020). Qorvo DWM3000 Datasheet. [Online]. Available: http:// www.DWM3000-Qorvo

[5] (2015). Qorvo DW1000 Datasheet. [Online]. Available: http:// www.DW1000-Qorvo

[6] R. Liu et al., "A 264- $\mu \mathrm{W}$ 802.15.4a-compliant IR-UWB transmitter in $22 \mathrm{~nm}$ FinFET for wireless sensor network application," in Proc. IEEE Radio Freq. Integr. Circuits Symp. (RFIC), Jun. 2018, pp. 164-167.

[7] G. de Streel et al., "Sleeptalker: A ULV 802.15.4a IR-UWB transmitter $\mathrm{SoC}$ in 28-nm FDSOI achieving $14 \mathrm{pJ} / \mathrm{b}$ at $27 \mathrm{Mb} / \mathrm{s}$ with channel selection based on adaptive FBB and digitally programmable pulse shaping," IEEE J. Solid-State Circuits, vol. 52, no. 4, pp. 1163-1177, Apr. 2017.

[8] J. Ryckaert, G. V. D. Plas, V. D. Heyn, C. Desset, B. V. Poucke, and J. Craninckx, "A 0.65-to-1.4 nJ/Burst 3-to-10 GHz UWB all-digital TX in $90 \mathrm{~nm}$ CMOS for IEEE 802.15.4a," IEEE J. Solid-State Circuits, vol. 42, no. 12, pp. 2860-2869, Dec. 2007.

[9] P. P. Mercier, D. C. Daly, and A. P. Chandrakasan, "An energy-efficient all-digital UWB transmitter employing dual capacitively-coupled pulseshaping drivers," IEEE J. Solid-State Circuits, vol. 44, no. 6, pp. 1679-1688, Jun. 2009.

[10] N.-S. Kim and J. M. Rabaey, "A high data-rate energy-efficient triple-channel UWB-based cognitive radio," IEEE J. SolidState Circuits, vol. 51, no. 4, pp. 809-820, Apr. 2016, doi: 10.1109/JSSC.2015.2512934.

[11] G. Lee, J. Park, J. Jang, T. Jung, and T. W. Kim, "An IR-UWB CMOS transceiver for high-data-rate, low-power, and short-range communication," IEEE J. Solid-State Circuits, vol. 54, no. 8, pp. 2163-2174, Aug. 2019, doi: 10.1109/JSSC.2019.2914584.

[12] S. Lanzisera, D. Zats, and K. S. J. Pister, "Radio frequency timeof-flight distance measurement for low-cost wireless sensor localization," IEEE Sensors J., vol. 11, no. 3, pp. 837-845, Mar. 2011, doi: 10.1109/JSEN.2010.2072496.

[13] J. Pandey and B. P. Otis, "A sub-100 $\mu$ W MICS/ISM band transmitter based on injection-locking and frequency multiplication," IEEE $J$. Solid-State Circuits, vol. 46, no. 5, pp. 1049-1058, May 2011, doi: 10.1109/JSSC.2011.2118030.

[14] B. Hong and A. Hajimiri, "A phasor-based analysis of sinusoidal injection locking in LC and ring oscillators," IEEE Trans. Circuits Syst. I, Reg. Papers, vol. 66, no. 1, pp. 355-368, Jan. 2019, doi: 10.1109/TCSI.2018.2860045.

[15] S. Yang, J. Yin, P.-I. Mak, and R. P. Martins, "A 0.0056-mm² -249-dB-FoM all-digital MDLL using a block-sharing offset-free frequency-tracking loop and dual multiplexed-ring VCOs," IEEE $J$. Solid-State Circuits, vol. 54, no. 1, pp. 88-98, Jan. 2019, doi: 10.1109/JSSC.2018.2870551.

[16] H. W. Pflug, "UWB pulse shaping for IEEE 802.15.4a," in Proc. 38th Eur. Microw. Conf., Amsterdam, The Netherland, Oct. 2008, pp. 713-716, doi: 10.1109/EUMC.2008.4751552.

[17] L. J. Paciorek, "Injection locking of oscillators," Proc. IEEE, vol. 53, no. 11, pp. 1723-1727, Nov. 1965, doi: 10.1109/PROC.1965. 4345.

[18] B. Mesgarzadeh and A. Alvandpour, "A study of injection locking in ring oscillators," in Proc. IEEE Int. Symp. Circuits Syst., Kobe, Japan, vol. 6, May 2005, pp. 5465-5468, doi: 10.1109/ISCAS.2005. 1465873 .
[19] S. Y. Lee, H. Ito, N. Ishihara, and K. Masu, "A novel direct injectionlocked QPSK modulator based on ring VCO in $180 \mathrm{~nm}$ CMOS," IEEE Microw. Wireless Compon. Lett., vol. 24, no. 4, pp. 269-271, Apr. 2014, doi: 10.1109/LMWC.2014.2299534.

[20] Q. van den Brande, S. Lemey, J. Vanfleteren, and H. Rogier, "Highly efficient impulse-radio ultra-wideband cavity-backed slot antenna in stacked air-filled substrate integrated waveguide technology," IEEE Trans. Antennas Propag., vol. 66, no. 5, pp. 2199-2209, May 2018, doi: 10.1109/TAP.2018.2809626.

[21] S. Agneessens, S. Lemey, T. Vervust, and H. Rogier, "Wearable, small, and robust: The circular quarter-mode textile antenna," IEEE Antennas Wireless Propag. Lett., vol. 14, pp. 1482-1485, 2015, doi: 10.1109/LAWP.2015.2389630.

[22] G. Quintero, J. F. Zurcher, and A. K. Skrivervik, "System fidelity factor: A new method for comparing UWB antennas," IEEE Trans. Antennas Propag., vol. 59, no. 4, pp. 2502-2512, Jul. 2011, doi: 10.1109/TAP.2011.2152322.

[23] Y. He et al., "24.7 A $673 \mu \mathrm{W} 1.8$-to- $2.5 \mathrm{GHz}$ dividerless fractional-N digital PLL with an inherent frequency-capture capability and a phasedithering spur mitigation for IoT applications," in IEEE Int. Solid-State Circuits Conf. (ISSCC) Dig. Tech. Papers, Feb. 2017, pp. 420-421, doi: 10.1109/ISSCC.2017.7870440.

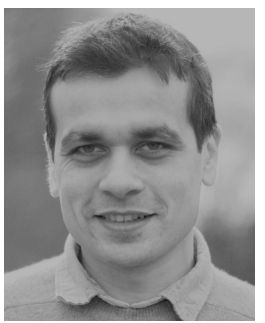

Gaurav Singh received the B.Tech. degree from DAIICT, Gandhinagar, India, in 2007, and the M.Sc. degree (cum laude) in microelectronics from Delft University of Technology, Delft, The Netherlands, in 2011.

From 2007 to 2009, he was working with Cypress Semiconductor, India, as an Application Engineer, defining next generation memory and mixed-signal programmable systems on chip products. From 2013 to 2019, he was with Qorvo, The Netherlands, where he worked on research and design of ultra-low-power radio SoCs for IoT applications. He joined Interuniversity Microelectronics Centre (IMEC), The Netherlands, in 2019, where he is leading the research in innovative ultra-low power wireless systems for sensing and communication in IoT and biomedical applications.

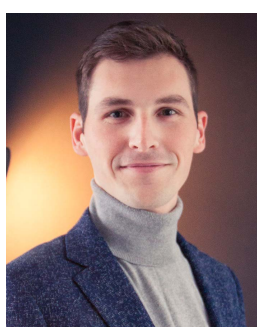

Erwin Allebes joined IMEC as an RF Researcher, in 2019, where he is currently involved in UWB radios and injection-locked frequency synthesizers. His research interests include ultra-low-power circuit design for IoT applications, digital PAs, and frequency synthesizers/clock generators.

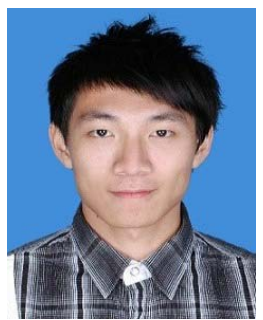

Yuming He received the B.S. degree in optical information and technology from South China University, Guangzhou, China, in 2012, and the M.S. degree in electronics engineering from Delft University of Technology, Delft, The Netherlands, in 2014.

Since 2015, he joined IMEC, The Netherlands, as a Researcher. $\mathrm{He}$ is interested in ultra-low-power digital PLL and mixed-signal IC design for IoT applications. 


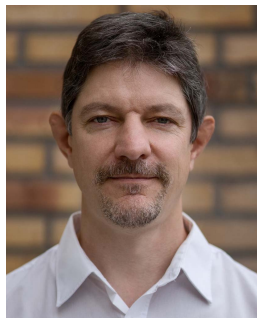

Evgenii Tiurin started his professional career in 1994 as an Electronics Engineer. As such, he worked for various research and development centres and production companies in Russia, Sweden, South Korea, and The Netherlands, mostly focusing on digital and mixed-signal ASICs for wireless communication and networking applications. Since 2015, he has been working with IMEC-Netherlands, Eindhoven, The Netherlands, as a Digital ASIC Design and Verification Engineer.

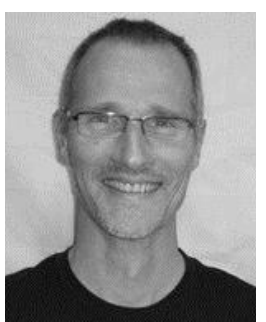

Paul Mateman received the bachelor's degree in computer technology from the Poly Technical School, Eindhoven, The Netherlands, in 1988, and the M.S. degree in electrical engineering from Delft University of Technology, Delft, The Netherlands, in 1993.

He has worked for Philips Research, Rockwell, NXP, ST Ericsson, and nVidia. He joined the Holst Centre/IMEC in 2014, where he works on mixed signal RF blocks (e.g., ADPLL).

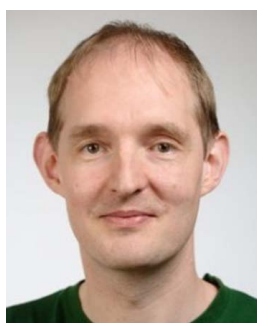

Johan F. Dijkhuis (Member, IEEE) received the M.S. degree in electrical engineering from the University of Twente, Enschede, The Netherlands, in 1998.

In 1998, he has worked as a RF and Analog Design Engineer for Philips Semiconductor, NXP, ST-Ericsson, and NVIDIA. Since 2014, he has been working with the Holst Centre/IMEC-Netherlands His research interests are ultra-low-power RF circuit design and power management circuits for ultra-lowpower radios.

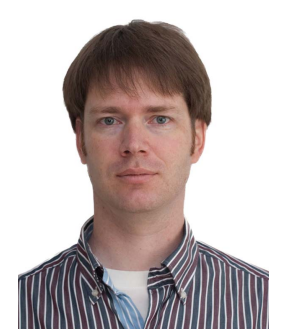

Gert-Jan van Schaik received the bachelor's degree from The Hague University of Applied Sciences, Hague, The Netherlands, in 1995.

He has worked for Agere Systems, Motorola B.V., and joined the Holst Centre/IMEC in 2011. He mainly works on digital design for digital base bands and neuromorphic computing. For several project, he also works as a technical lead and/or project lead.

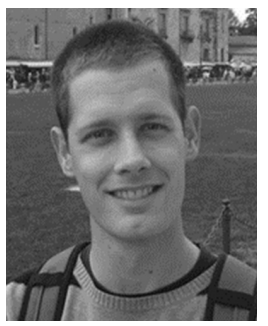

Elbert Bechthum received the Ph.D. degree (cum laude) from Eindhoven University of Technology, Eindhoven, The Netherlands, in 2015.

$\mathrm{He}$ is currently working in IMEC on ultra-lowpower radios and cellular IoT. His research interests include high performance data converters, low power radios, digital PLLs, and digital PA's.

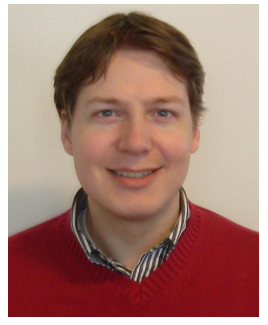

Johan van den Heuvel was born in Oss, The Netherlands, in February 1981. He received the M.Sc. and Ph.D. degrees in electrical engineering from the Eindhoven University of Technology, Eindhoven, The Netherlands, in 2006 and 2012, respectively.

He works at IMEC-Netherlands as the Wireless Research Scientist. His current research interests include all digital phase locked loops, low-power and high-speed mixed signal circuits and architectures, multiple antenna systems, ultra wideband communication, channel measurements, and digital baseband algorithms.

Dr. Heuvel received the "Veder Prijs 2012" for his Ph.D. thesis.

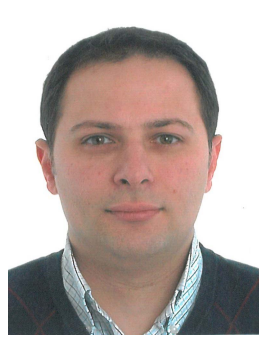

Mohieddine El Soussi (Member, IEEE) received the M.Sc. degree in communication engineering from the Technische Universität München, Munich, Germany, in 2007, and the Ph.D. degree from the Université Catholique de Louvain (UCLouvain), Louvain-la-Neuve, Belgium, in 2014.

Since 2016, he has been with IMEC, Eindhoven, The Netherlands. He was involved in various wireless communication solutions for NB-IoT, Bluetooth LE, UWB, and others. His research interests include cooperative networks, physical layer security, MIMO, localization, network coding, energy-efficient transceivers, and optimization.

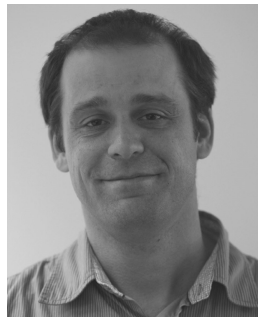

Arjan Breeschoten received the B.Sc. degree in computer technology in 1994.

Until 2008, he has worked within the semiconductor industry involved in research and development for ASICs in the security, WiFi, WiMax, and DECT application domains. In 2009, he joined the Holst Centre/IMEC, Eindhoven, The Netherlands, where he focuses on system integration and project management for ultra-low-power communications solutions.

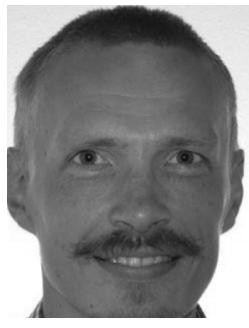

Hannu Korpela received the bachelor's degree from Central Ostrobothnia Polytechnic, Ylivieska, Finland, in 2000

$\mathrm{He}$ has excellent knowledge from RF measurements during his 20 years long career and has a good understanding of various measurement instruments. He also has experience with automating RF measurements using Python. From 2000 to 2007, he was an RF Test Engineer with Nokia, Salo, Finland, where he tested the first wideband code division multiple access (WCDMA) mobile phone in the world. From 2007 to 2013, he was a Senior Test Engineer with ST-Ericsson, Turku, Finland. From 2014 to 2021, he was a Radio Evaluation Engineer with IMEC Netherlands, Eindhoven, The Netherlands. Since 2021, he has been a Project Engineer with Cencorp Automation, Salo. 


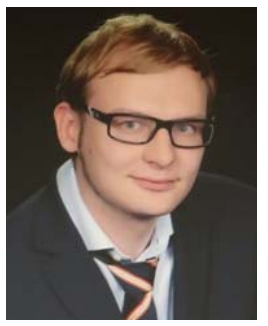

Gert-Jan Gordebeke (Member, IEEE) was born in 1995. He received the B.Sc. and M.Sc. degrees in electrical engineering from Ghent University, Ghent, Belgium, in 2015 and 2017, respectively, where he is currently pursuing the Ph.D. degree in electrical engineering with IDLab, Electromagnetics Group, Department of Information Technology, IMEC.

His current research interests include antenna design using unconventional substrates and robust antenna systems for ultra-wideband localization.

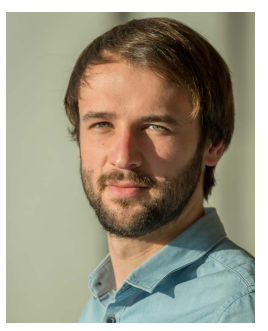

Sam Lemey (Member, IEEE) received the M.Sc. degree in electronic engineering from Howest, University College West Flanders, Kortrijk, Belgium, in 2012, and the Ph.D. degree in electrical engineering from Ghent University, Ghent, Belgium, in 2016.

$\mathrm{He}$ is currently an Assistant Professor with Ghent University and a Post-Doctoral Fellow of the Special Research Fund (BOF), affiliated with the IDLabElectromagnetics Group, Department of Information Technology (INTEC), Ghent University/imec. From January to March 2018, he was the Visiting Scientist of the Terahertz Photonics Group, Institute of Electronics, Microelectronics and Nanotechnology (IEMN), University Lille Nord de France, Lille, France. His research interests include antenna systems for wearable applications, active antenna design for the Internet of Things and (beyond) 5G applications, (opto-electronic) millimeter-wave multi-antenna systems, impulse-radio ultrawideband antenna systems for centimeter-precision localization, and fullwave/circuit co-optimization frameworks to realize (opto-electronic) active (multi-)antenna systems.

Dr. Lemey received the URSI Young Scientist Award at the 2020 URSI General Assembly and was awarded the Best Paper Award at the 2016 IEEE MTT-S Topical Conference on Wireless Sensors and Sensor Networks. He was also a co-recipient of the 2015 Best Paper Award at the 22nd IEEE Symposium on Communications and Vehicular Technology in the Benelux and the 2019 ECOC Best Demo Award.

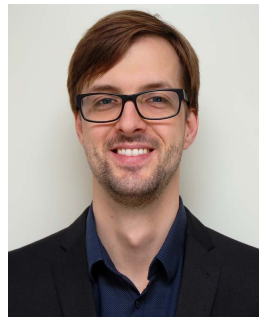

Christian Bachmann (Member, IEEE) received the $\mathrm{Ph} . \mathrm{D}$. degree in electrical engineering from Graz University of Technology, Graz, Austria, in 2011, in collaboration with Infineon Technologies.

He joined IMEC in 2011, working on ultra-low-power DSP and wireless communication systems, digital baseband (DBB) processing, and hardware/software co-design. He is currently the Program Manager of IMEC's UWB and Bluetooth Secure Proximity programs, addressing research and development challenges for high-accuracy, secure wireless localization with Bluetooth, as well as ultra-wideband (UWB) and for advanced ultra-low power (ULP) RF wireless transceiver circuits enabling next-generation automotive, medical, and the IoT applications.

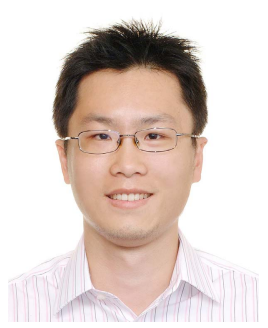

Yao-Hong Liu (Senior Member, IEEE) received the Ph.D. degree from National Taiwan University, Taipei, Taiwan, in 2009.

He was with Terax, Via Telecom (now Intel), and Mobile Devices, Taiwan, from 2002 to 2010, working on Bluetooth, WiFi, and cellular wireless $\mathrm{SoC}$ products. In 2010, he joined IMEC, Netherlands, where he has been leading the research development of the ultra-low power wireless ASIC design. He is currently a Principal Member of Technical Staff at IMEC. His research focus is on high-capacity and minimally-invasive wireless technology for implantable neural interfaces.

Dr. Liu has served on a Technical Program Committee of IEEE ISSCC, and currently serves in IEE RFIC Symposium. He was a recipient of the European Research Council (ERC) Personal Grant and is the Principal Investigator of "Intranet of Neurons." He was a recipient of the ISSCC 2020 Best System Demonstration Award. 